\title{
Special Section Guest Editorial: Instrument Calibration and Product Validation of GOES-R
}

\author{
Xiangqian Wu, Changyong Cao, Satya Kalluri, and Jaime Daniels \\ National Oceanic and Atmospheric Administration, National Satellite, Data, and Information \\ Service, Center for Satellite Applications and Research, College Park, Maryland, United States
}

The first Geostationary Operational Environmental Satellite (GOES) was launched in October 1975, five years after the establishment of the National Oceanic and Atmospheric Administration (NOAA). The first GOES of the second generation (GOES-8) was launched in April 1994.

The demands for and the technologies to acquire new and improved environment data from geostationary platforms have increased tremendously in the past two decades. GOES-R, the third generation of GOES, is NOAA's response to those demands. Compared to the imager instrument on the second generation of GOES, the Advanced Baseline Imager (ABI) on GOES-R has three times more spectral channels, four times better spatial resolution, five times faster refresh rate, and much improved data quality. The Geostationary Lightning Mapper (GLM) is the first ever on a geostationary platform that offers a unique way of monitoring the evolution of weather. GOES$\mathrm{R}$ also carries a suite of space weather instruments, including Extreme Ultraviolet and X-ray Irradiance Sensor (EXIS), Magnetometer (MAG), Space Environment In-Situ Suite (SEISS), and Solar Ultraviolet Imager (SUVI). These new and improved capabilities of the third generation of GOES are critical for NOAA's mission of protecting lives and property by providing continuous surveillance of the land, ocean, and atmosphere of the United States and its surrounding environment.

GOES-16 was the first of the new generation GOES. It was launched in November 2016 and has been operational as GOES-EAST since December 2017; GOES-17 was launched in March 2018 and has been operational as GOES-WEST since January 2019. After the successful deployment of GOES-R instruments and dissemination of their products, the Journal of Applied Remote Sensing documents in this special section some of the works on instrument calibration and product validation of GOES-R sensors. Gibbs et al. described the theoretical basis of tuning Kalman filter with maximum likelihood estimation, which is the foundation of ABI Image Navigation and Registration (INR). Carr et al. and Tan et al. discussed the validation of INR for GLM and ABI, respectively. Peterson et al. analyzed three ways to remove solar artifacts from GLM data. Bateman and Mach assessed the detector efficiency and false alarm rate of GLM calibration. Caron and Rollins demonstrated how to improve the uniformity of lunar images to obtain more accurate modulation transfer function (MTF). Cook et al. proposed a method to alleviate $\mathrm{ABI}$ image striping. Uprety et al. and Bhatt et al. validated the calibration of ABI solar reflectance bands. Gunshor et al. documented a number of ABI calibration and operation deficiencies, some were in the past and some may still be on-going. Collectively, these papers cover the radiometric and geometric calibration and validation of ABI and GLM.

We would like to take this opportunity to thank the authors, reviewers, and the JARS editorial team, who made this special section possible. We regret that this special section cannot accommodate some of the papers on GOES-R instrument calibration and product validation, and the published papers did not cover the space weather instruments. We are confident, however, that this special section will inspire renewed interest in satellite instrument calibration.

(C) 2020 Society of Photo-Optical Instrumentation Engineers (SPIE) 